\title{
ESTRUCTURA Y ECOLOGÍA DE LA POBLACIÓN DEL ENDEMISMO CUBANO BROUGHTONIA CUBENSIS (ORCHIDACEAE), EN CABO SAN ANTONIO, PENÍNSULA DE GUANAHACABIBES, PROVINCIA DE PINAR DEL RÍO, CUBA
}

\author{
Elaine GonZÁlez HeRnÁNDEZ ${ }^{1}$, JoseP RAVENTÓs ${ }^{2}$, \\ ERnesto Múuica BeníteZ ${ }^{1,3} \&$ Andreu Bonet ${ }^{2}$ \\ 'Jardín Botánico Orquideario Soroa, Apdo. Postal No.5. Candelaria. Pinar del Río. Cuba. \\ ${ }^{2}$ Departamento de Ecología. Universidad de Alicante. Apdo. Postal No.99, 03080 Alicante. España. \\ ${ }^{3}$ Autor para correspondencia: emujica@vrect.upr.edu.cu
}

\begin{abstract}
We analyze some structural characteristics of a population of Broughtonia cubensis, an epiphytic orchid endemic to Cuba, in Guanahacabibes peninsula, Pinar del Rio province, involving ten transects. This species showed an aggregate distribution with a high coespecific competition. It is selectively found on Plumeria tuberculata and Erythroxylum areolatum phorophytes. They are found predominately on individuals with smooth or rugose barks and in north and east orientation. The quality hypothesis of sites is discussed in relation to the possible presence of micorrhizae. Finally, inside the area included by the 10 transects, this species has a metapopulational structure with a nuclear zone located in the area between the semidecidous forest and the marsh vegetation.
\end{abstract}

RESUMEN: Se analizan algunas características de la estructura poblacional de Broughtonia cubensis, orquídea epífita endémica de Cuba, en Cabo San Antonio, península de Guanahacabibes, provincia de Pinar del Río, mediante el análisis de diez transectos. Esta especie presenta una distribución agregada con una alta competencia intraespecífica. Además, muestra una elevada selectividad en la especie de forofito sobre la que habita, presentando una marcada preferencia por Plumeria tuberculata y Erythroxylum areolatum. Predomina la presencia de la especie sobre corteza lisa o rugosa y en orientación norte y este. Se discute la hipótesis de la calidad de sitios en relación a la posible presencia de micorrizas. Finalmente, dentro del área abarcada por los 10 transectos, se ha encontrado que esta especie tiene una estructura metapoblacional con una zona núcleo localizada entre el bosque semideciduo y la vegetación de pantano.

Palabras Claves / Key wORDS: Orchidaceae, Broughtonia cubensis, ecología, clases de vida, competencia, forofitos, Cuba.

\section{Introducción}

La familia Orchidaceae contiene el número más grande de especies de cualquier familia del reino vegetal (ca. 25.000 especies) y ha recibido una gran atención debido a su enorme valor económico y social comparado con otras especies (Kindlmann et al. 2002). Esta familia es la más numerosa y probablemente la más evolucionada de todas las plantas, por lo que constituye un componente muy importante de la biodiversidad (Mújica et al. 2000). En relación a otras familias de plantas, hay evidencia de que las orquídeas están sujetas a un alto peligro de extinción ya sea por causas naturales o antropogénicas (Hutchings 1989). En años recientes muchas especies parecen estar en un nivel crítico pues las estrategias de conservación de las mismas son complicadas debido a su ciclo de vida complejo y no pueden llevarse a cabo sin un estudio poblacional. Sin embargo, la mayor parte de los estudios poblacionales sobre orquídeas se han limitado a especies terrestres [ver reseñas de Kull 2002, Kindlmann et al. 2002, número monográfico de Biological Conservation 129 (2006)] y pocos se han llevado a cabo en especies de orquídeas epífitas o litófitas (Zotz \& Tyree 1996, Zotz 1998, 1999, 2000, Tremblay 1997, Tremblay \& Hutchings 2003).

Las orquídeas están consideradas entre las familias mejor representadas en la flora vascular cubana (Díaz 1996), distribuidas a través de todo el archipiélago con un 30,5\% de especies endémicas (Mújica, 
inédito). Broughtonia cubensis es una especie epífita endémica de Cuba, y se ha considerado siempre como emblemática de la flora orquideológica cubana, de ahí que siempre ha sido motivo de preocupación su estado de conservación por parte de botánicos cubanos y foráneos. En el presente estudio se ha llevado a cabo una evaluación preliminar de los aspectos de la ecología y dinámica de las poblaciones de esta especie, teniendo en cuenta su estructura poblacional en clases de vida, en condiciones aisladas y en condiciones agregadas. Este estudio ha de servir de base para la planificación de una estrategia de manejo sostenible de este endemismo epífito cubano que presenta una estructura metapoblacional. Esta formado por tres subpoblaciones, una pequeña población en Loma La Coca, provincia de La Habana, donde está en peligro de extinción por las alteraciones en el hábitat debido a la presión urbanística y a la depredación y dos poblaciones en áreas protegidas de la Reserva de la Biosfera Península de Guanahacabibes. El presente estudio se localiza en las zonas de Playa El Francés, y en los alrededores del Faro Roncali, en Cabo San Antonio, ocupando en esta área el matorral xeromorfo costero.

Con el mismo nos proponemos contestar a las siguientes preguntas:

- ¿Es Broughtonia cubensis (B.c.) una especie especialista o generalista en la elección del forofito?

- ¿Esta especie muestra alguna preferencia en el tipo de corteza asociada al forofito?

- ¿Cuál es el patrón de distribución espacial de esta especie?

- ¿Hay algún tipo de zonación vertical en la distribución de B.c. a lo largo del forofito?

- ¿Es la competencia intra e interespecífica una interacción importante para esta especie?

- ¿Cual es el patrón de distribución espacial para las distintas formas de vida en B.c.?

\section{Materiales y métodos}

Área de estudio - La Reserva de la Biosfera Península de Guanahacabibes (R.B.P.G.), se ubica en el extremo más occidental de la isla de Cuba, específicamente en el municipio Sandino, perteneciente a la provincia de Pinar del Río. Limita al N con el Golfo de México, al S con el Mar Caribe, al E con la demarcación vial desde La Fe hasta Manuel Lazo y al W con el estrecho de Yucatán (Fig. 1). Es un territorio

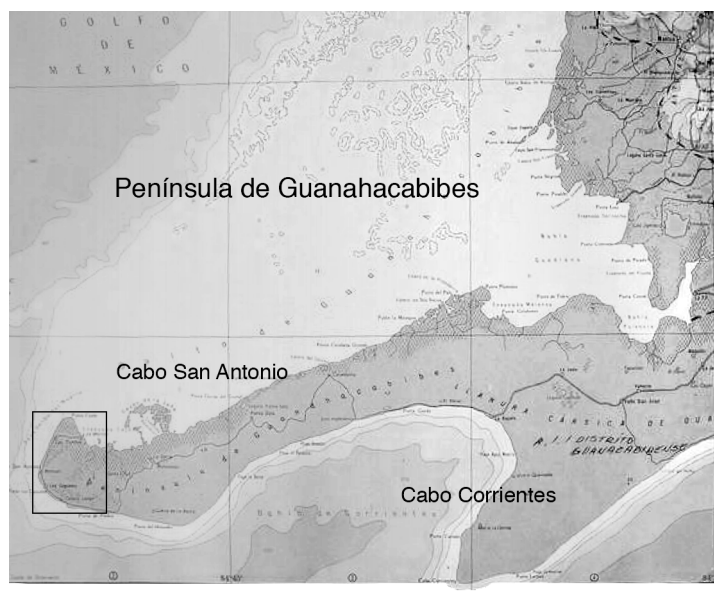

Figura 1. Península de Guanahacabibes con ambos cabos y área de estudio.

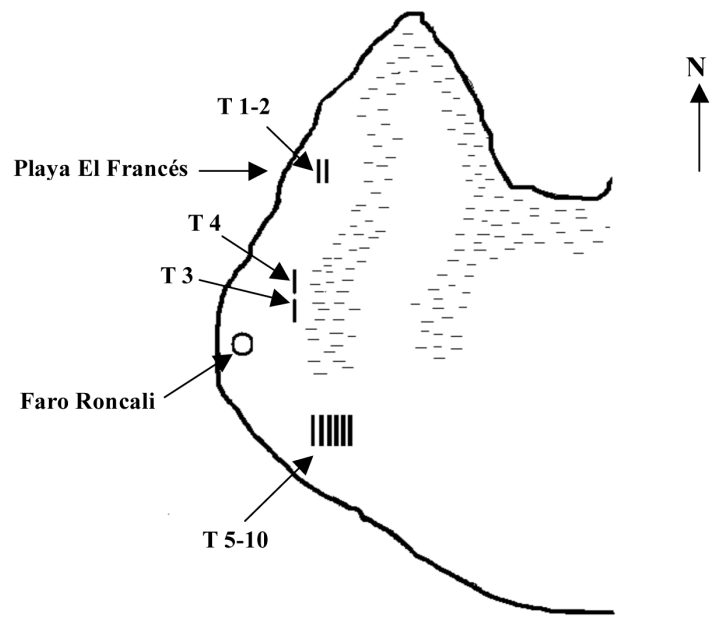

FiguRA 2. Área de trazados de transectos en Cabo San Antonio.

alargado, estrecho y llano de unas 101500 hectáreas (Herrera et al. 1987), de las cuales 28000 comprenden el área terrestre del parque, dos ubicadas en la península de Guanahacabibes, una de las cuales es objeto de estudio actualmente.

Para efectuar el presente estudio se procedió al trazado de transectos. Los mismos se trazaron tanto paralelos, como perpendiculares a la línea de la costa para establecer los límites de dicha población (Fig. 2). Esta especie se localiza básicamente sobre el matorral xeromorfo costero, en una estrecha franja sobre una losa de diente de perro (limestone) que raramente supera los $20 \mathrm{~m}$. de ancho. Es por ello que se decidió trazar transectos de $5 \mathrm{~m}$ de ancho $\mathrm{x} 100 \mathrm{~m}$ de longitud, dejando otros $5 \mathrm{~m}$ entre uno y otro, tra- 
tando de abarcar la mayor área posible de esta franja, pues en el bosque semideciduo es rara (Ferro, com. pers.).

Se trazaron un total de 10 transectos tratando de delimitar la población y reunir en ellos la mayor cantidad de individuos posibles. Los dos primeros (T-1 y T-2) se trazaron cerca de la costa oeste, con orientación S-N, en la zona de playa El Francés, paralelos a la misma, en el matorral xeromorfo costero, lo que por el momento es el límite $\mathrm{N}$, teniendo al $\mathrm{E}$ una zona pantanosa y al $\mathrm{W}$ un estrecho sistema de vegetación de costa arenosa, en los cuales no habita. Al $\mathrm{N}$ y $\mathrm{S}$ el bosque semideciduo donde es rara su presencia. Los T-3 y T-4 fueron trazados a $1.5 \mathrm{~km}$ al $\mathrm{S}$ de los primeros en una faja de unos 20 $\mathrm{m}$. de ancho entre el bosque semideciduo y la vegetación de pantano con igual orientación y límites a los anteriores. El resto, del T-5 al T-10, se trazaron a $1 \mathrm{~km}$. al sureste de los anteriores, con igual orientación pero casi perpendiculares a la costa sur, teniendo como límite $\mathrm{S}$ la vegetación de costa arenosa y en las otras tres direcciones el bosque semideciduo en los cuáles como hemos dicho, está prácticamente ausente. Todo parece indicar que el T-10 se encuentra ya en el límite E de la población. Por el momento el área ocupada se estima en $13000 \mathrm{~m}^{2}$.

En relación al clima, la península difiere poco del resto del territorio de Cuba occidental, aunque su forma alargada y estrecha hace que sea más afectada por el mar que la rodea y la corriente del Golfo de México. La precipitación anual promedio es de 1334 mm (Lopetegui et al. 1999), la cual no es uniforme si tenemos en cuenta los promedios de su parte meridional y septentrional que son de 1000-1200 $\mathrm{mm}$ y $1400-1600 \mathrm{~mm}$ respectivamente. La humedad relativa permanece casi invariable con un $81 \%$ de promedio (Lopetegui et al. 1999). Los promedios anuales de temperatura mínima y máxima son de $21.5^{\circ} \mathrm{C}$ y $29.2^{\circ} \mathrm{C}$. La dominancia todo el año de los vientos del nordeste es latente. El período de sequía se extiende desde noviembre hasta abril.

\section{CARACTERÍsticas de Broughtonia CUBENSIS -} Broughtonia cubensis presenta usualmente individuos de mediano a pequeño tamaño con 1-2 hojas en cada pseudobulbo. Sus flores son vistosas, con excelente fragancia, pequeñas, con sépalos y pétalos blancos con labelo también blanco y callo amarillento-purpúreo. Florece mayormente en enero, exten- diéndose hasta marzo. Entre un $25-30 \%$ de sus flores concebían frutos en áreas bajo monitoreo antes del paso del huracán Iván en septiembre del 2004. Sus frutos liberan las semillas en unos 40 días (Mújica, inédito).

En la R.B.P.G. podemos encontrar una decena de diferentes formaciones vegetales donde se concentran 14 especies endémicas de la misma. El bosque semideciduo es el predominante en toda su extensión, principalmente sobre los suelos cársicos de la parte central. En la península se han identificado un total de 750 especies vegetales, de las cuales 500 son de amplia distribución, 231 endémicos y 19 endémicos distritales (Urquiola, com. pers.). B.c. se encuentra en el bosque semideciduo y en el matorral xeromorfo costero, siendo rara su presencia en el primero. El suelo está compuesto por el carso desnudo conocido como "diente de perro", en ambientes abiertos a plena exposición solar.

B.c. es una especie que además de encontrarse en esta península se le reporta también para algunos puntos de la costa norte de la provincia Habana donde ya está prácticamente extinguida por la depredación y el aumento cada vez mayor del uso de los terrenos donde se encuentra con fines urbanísticos.

En la zona donde habita B.c., hemos encontrado otras 8 especies de orquídeas que viven simpátricamente. Para estudiar el efecto de la competencia se marcaron e identificaron todos los individuos que estuvieran a un radio menor de $10 \mathrm{~cm}$ de cada planta de B.c. Se ha determinado este radio por estimarse que a uno mayor la competencia no es significativa de acuerdo al tamaño usualmente pequeño de los individuos de esta especie, además se tuvo en cuenta la distancia real al vecino más cercano para establecer la competencia por el hábitat y la especie de ese vecino, determinando así, aquellas más representativas y establecer un análisis comparativo para conocer la posible incidencia de la competencia por el hábitat en el cambio anual de la estructura de las clases poblacionales, y en el área foliar, parámetro que se decidió escoger para delimitar las clases tal y como se explica a continuación.

Las clases de vida - Se clasificó la población según sus formas de vida en 4 clases: plántulas (Clase 1), juveniles (Clase 2), adulto reproductor I (Clase 3), adulto reproductor II (Clase 4). Al no haber podido encontrar estudios publicados de poblaciones estruc- 
turadas en orquídeas epífitas tropicales se delimitaron las clases mediante aproximaciones sucesivas. Primero se clasificó a los individuos en dos grandes grupos: reproductores y no reproductores. Para posteriormente sub-dividirlos en clases, se utilizó como parámetro el área foliar, pues existe una relación positiva entre área y tamaño. Para esto se contaron en cada individuo el total de hojas midiéndose el largo por ancho de cada una de ellas para luego calcular el promedio por cada ejemplar.

Se consideró también que esta especie es muy variable en tamaño, existiendo individuos muy pequeños ya en capacidad reproductiva y por el contrario, otros de mayor tamaño aún en estado juvenil. Para subdividir a los reproductores se calculó el área foliar promedio de todos los individuos de este grupo, llegando a ser de $12 \mathrm{~cm}^{2}$. Por tanto, se decidió que todos los que estuvieran por encima de este número entrarían en la clase C-4, mientras los que presentaron un área menor conformarían la clase C-3. La división en clases de los no reproductores se hizo más difícil. Para ello se tuvieron en cuenta criterios de observación, las plántulas son minúsculas en comparación con el resto de las clases. Al comparar in-situ las áreas foliares y tamaños de las plántulas surgidas el último año, se comprobó que estas no sobrepasaban el $1,5 \mathrm{~cm}^{2}$ de área foliar (Mújica, inédito), por lo cual todos los individuos con área foliar inferior o igual a esta serían catalogados como plántulas, o sea $\mathrm{C}$ - 1 , mientras los que presentaran un área mayor conformarían la clase de juveniles ó C-2.

Una vez que se determinaron las clases de vida, se dividieron los individuos en dos grupos, los que se encuentran en condiciones aisladas y los que se encuentran en condiciones agregadas. Con el estudio de este parámetro queremos detectar en el caso de los individuos agregados el tipo de competencia que se establece entre B.c. y las especies de orquídeas vecinas. Seguidamente se realizó el conteo de los individuos en clases de vida en condiciones aisladas y agregadas, como se describió anteriormente.

\section{Análisis estadísticos}

Una aproximación para ver si las especies de forofitos están distribuidas al azar o agregadas es aplicar una distribución de Poisson. Está distribución tiene la varianza igual a la media. Si este cociente entre la varianza y la media (coeficiente de dispersión, CD) está cercano a " 1 " los individuos están repartidos al azar, si es mayor que 1, están agregados y si es menor que 1 están distribuidos regularmente. El test de Chicuadrado permite comparar las distribuciones esperadas teóricamente de las obtenidas y así determinar el grado de agregación.

Para ver si hay una relación entre las características del forofito y la presencia o no de B.c. hemos usado el test de Chi-cuadrado para ver si había asociación entre las variables categóricas (Sokal \& Rohlf 1981).

\section{Resultados}

DIVERSIDAD DE FOROFITOS OCUPADOS A LO LARGO DE LOS TRANSECTOS - La figura 3 muestra un gráfico de rango-abundancia de forofitos ocupados por B.c. en los 10 transectos realizados. Se observa que las curvas en los transectos indican una estructura diferente. En el T-2 se muestra una mayor equitatividad en la ocupación de especies de forofitos por parte de B.c. Contrariamente en T-7 y T-4 se encontró una presencia muy marcada de Plumeria tuberculata. El rango muestra asimismo una indicación de la riqueza en cada transecto, presentando T-2 y T-7 una mayor riqueza de especies de forofitos ocupados.

Se debe destacar que, en el caso de B.c., un total de 14 especies de forofitos han resultado ocupadas al menos con un individuo, sin embargo, como se apreciará más adelante, la especie en estudio tiene sus preferencias por algunos de ellos.

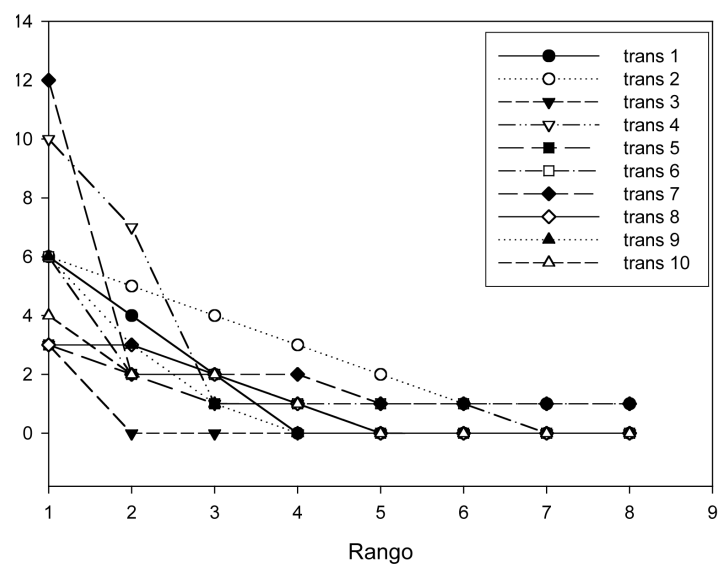

Figura 3. Diversidad de forofitos ocupados. Rango-abundancia en los 10 transectos. 


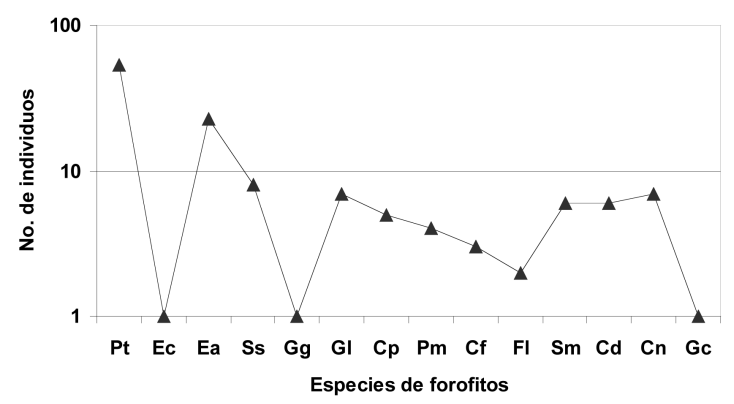

Figura 4. Número de forofitos ocupados de cada especie en los 10 transectos.

DisTRIBUCIÓN DE FOROFITOS OCUPADOS - La figura 4 muestra que la comunidad de forofitos ocupados es diversa, sin embargo, se observa una preferencia por Plumeria tuberculata $(\mathrm{Pt})$ y Erythroxylum areolatum (Ea). De los 127 forofitos ocupados el $42 \%$ corresponden a Plumeria tuberculata y el $18 \%$ a Erythroxylum areolatum. Entre ambas representan el $60 \%$ de todos los forofitos ocupados.

Aunque el estudio no perseguía el objetivo de conocer la abundancia de todas las especies de forofitos, sí podemos decir que son estas las dos especies de forofitos claves sobre las cuáles vive la población en el área estudiada. Existe una diferencia significativa entre ocupar estas dos especies y las demás. Otras especies sumamente abundantes en el área estudiada presentaron, por el contrario, muy bajos por cientos

TABLA 1. Matriz de similitudes antre los 10 transectos.

\begin{tabular}{|c|c|c|c|c|c|c|c|c|c|}
\hline & 1 & 3 & 4 & 5 & 6 & 7 & 8 & 9 & 10 \\
\hline 1$]^{*}$ & 68,571 & 0 & 38,71 & 31,579 & 64 & 35,294 & 28,571 & 54,546 & 19,048 \\
\hline $2^{*}$ & * & 23,077 & 52,381 & 33,333 & 50 & 44,444 & 37,5 & 42,424 & 43,75 \\
\hline 3 * & * & * & 27,273 & 20 & 0 & 8 & 33,333 & 0 & 50 \\
\hline 4 * & * & * & * & 23,077 & 37,5 & 53,659 & 35,714 & 41,379 & 42,857 \\
\hline 5 * & * & * & * & * & 30 & 27,586 & 37,5 & 23,529 & 37,5 \\
\hline 6 * & * & * & * & * & * & 57,143 & 45,455 & 60,87 & 27,273 \\
\hline $7{ }^{*}$ & * & * & * & * & * & * & 38,71 & 50 & 32,258 \\
\hline 8 * & * & * & * & * & * & * & * & 31,579 & 44,444 \\
\hline 9 * & * & * & * & * & * & * & * & * & 52,632 \\
\hline $10{ }^{*}$ & * & * & * & * & * & * & * & & \\
\hline
\end{tabular}

TABLA 2. Distribución de especies ocupadas por transectos.

\begin{tabular}{|l|r|r|r|r|r|l|}
\hline & Varianza & Media & Chi-sq & g.l. & Probabilidad & Agregación \\
\hline Especies & 13,7889 & 5,3 & 23,4151 & 9 & 0,0054 & Agregado \\
\hline Plumeria tuberculata & 0,1 & 0,1 & 9 & 9 & 0,4375 & al azar \\
\hline Enythroxylum confusum & 5,7889 & 2,3 & 22,6522 & 9 & 0,0071 & Agregado \\
\hline Enythroxylum areolatum & 2,8444 & 0,8 & 32 & 9 & 0,0002 & Agregado \\
\hline Stigmatophyllum sagraeanum (lianas) & 0,1 & 0,1 & 9 & 9 & 0,4375 & al azar \\
\hline Gerascanthus gerascanthoides & 0,9 & 0,7 & 11,5714 & 9 & 0,2378 & al azar \\
\hline Gymnantes lucida & 0,9444 & 0,5 & 17 & 9 & 0,0484 & al azar \\
\hline Catalpa pubescens & 0,9333 & 0,4 & 21 & 9 & 0,0127 & Agregado \\
\hline Pichrodendrom macrocarpum & 0,4556 & 0,3 & 13,6667 & 9 & 0,1339 & al azar \\
\hline Cithraxylum fruticosum & 0,1778 & 0,2 & 8 & 9 & 0,5351 & al azar \\
\hline Ficus laenigata & 0,4889 & 0,6 & 7,3333 & 9 & 0,6036 & al azar \\
\hline Secos o muertos & 1,1556 & 0,6 & 17,3333 & 9 & 0,0435 & al azar \\
\hline Conocladia dentata & 1,3444 & 0,7 & 17,2857 & 9 & 0,0442 & al azar \\
\hline Chascoteca neopeltandra & 0,1 & 0,1 & 9 & 9 & 0,4375 & al azar \\
\hline Getharda calyptrata & & & & & & \\
\hline
\end{tabular}

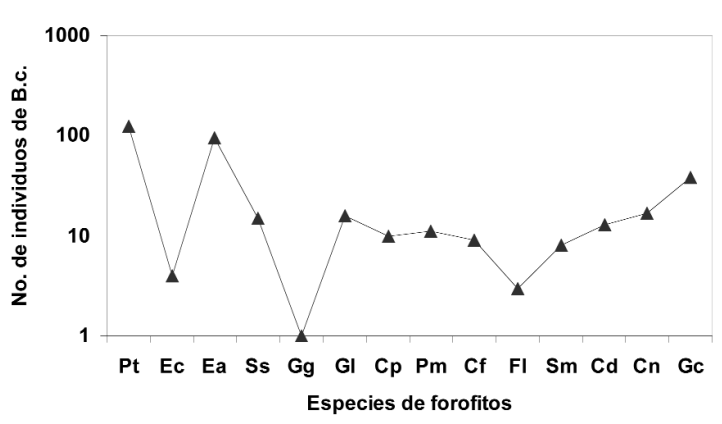

Figura 5. Número de individuos de B. cubensis en las diferentes especies de forofitos ocupados.

de ocupación, como por ejemplo: Gymnanthes lucida (Gl) con 5,5\%, Chascoteca neopeltandra (Cn) 5,5\%, Catalpa pubescens (Cp) 3,9\%, Citharexylum fruticosum (Cf) 2,3\% y Gerascanthus gerascanthoides $(\mathrm{Gg})$ $0,7 \%$. Otros como Stigmatophyllum sagraeanum (Ss), Conocladia dentata, Picrodendron macrocarpum, Citharexylum fruticosum (Cf), Ficus laevigata (F1), Secos o muertos $(\mathrm{Sm})$, presentaron valores medios y Guettarda calyptrata (Gc) con un solo forofito ocupado aunque no es una especie abundante.

Las similitudes o diferencias entre transectos, de acuerdo a las especies de forofitos ocupados, pueden claramente observarse en la matriz de similitudes entre ellos (tabla 1), a partir del índice de BrayCurtis. Los valores indican similitud en la composición de los forofitos ocupados en los transectos. A mayor valor, mayor similitud. Se observa elevada similitud entre los transectos T-1 con T-2 y T-6, así como T-6 con T-9, mientras el T-3 es el que presenta menor similitud global, pues no se relaciona con los T-1, T-6 y T-9.

La Tabla muestra que la mayor parte de los forofitos ocupados por B.c. se encuentran distribuidos al azar (10/14) aún en algunos casos indican agregación de especies, como en Plumeria tuberculata, Erythroxylum areolatum, Stigmatophyllum sagraeanum (Lianas) y Picrodendron macrocarpum.

Del total de individuos de B.c. localizados y marcados (365 ind.), el 33,9\% se encontró sobre Plumeria tuberculata (124 ind.), mientras que sobre Erythroxylum areolatum el 26,3\%, (96 ind.) para un $60,27 \%$ del total general (220 ind.) entre ambos. Otro forofito importante en cuanto a ocupación lo es Getharda calyptrata con el 10,4\% (38 ind.), aún cuando solo se encontró un solo individuo de esta especie de forofito ocupada (figura 5). 


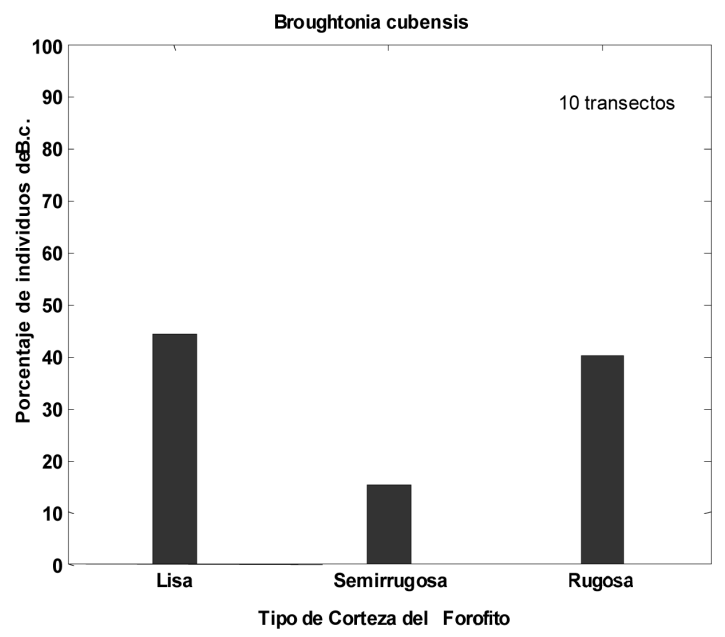

FiguRa 6. Tipo de corteza preferida por B. cubensis.

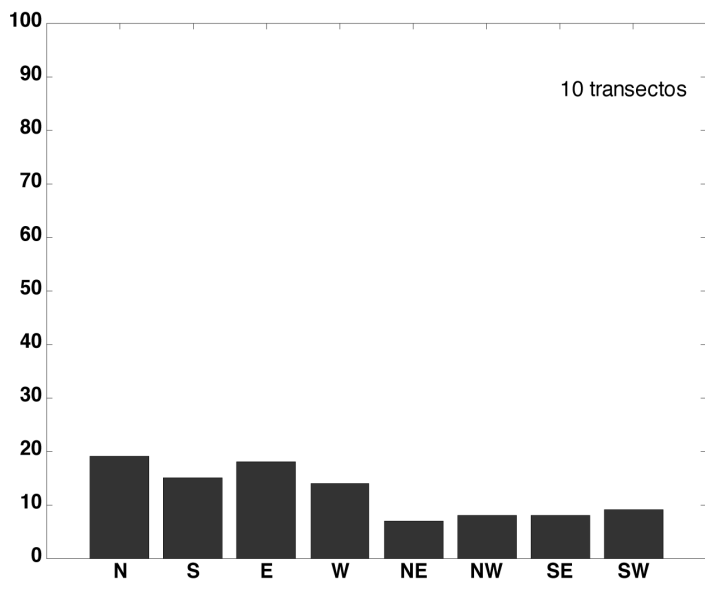

FIgURA 7. Orientación de B. cubensis.

TIPO DE CORTEZA DE LOS FOROFITOS OCUPADOS - La figura 6 muestra el porcentaje de individuos en relación al tipo de corteza del forofitos. El análisis de chicuadrado nos permite rechazar la hipótesis nula de que B.c. prefiere cualquier tipo de corteza por igual $(p<0.0001)$. De hecho podemos decir que prefiere la corte lisa o rugosa pero no la semirrugosa. Esto está determinado por ser Plumeria tuberculata (lisa) y Erythroxylum areolatum (rugosa) las especies ocupadas más comunes.

ORIENTACIÓN ESPACIAL - La figura 7 nos muestra la orientación espacial de los individuos en los 10 transectos estudiados, existiendo una tendencia marcada a ubicarse hacia el norte y hacia el este, siendo menor en el noreste, no todas las direcciones son igualmente probables $\left(\chi^{2}=47.775, \mathrm{p}=0.0001\right)$.

ANÁlisis DE LA COMUNIDAD DE EPÍFITAS - Un total de 9 especies de orquídeas pueden ser encontradas en el área monitoreada, incluyendo a $B$. cubensis $(\mathrm{Bc})$. Esta especie junto a Encyclia $\mathrm{sp}$. (Esp) son las más abundantes (existen tres especies del género Encyclia en el área de Cabo San Antonio, Encyclia bocourtii y Encyclia plicata en la zona de los T-1 al T-4 y Encyclia fucata y Encyclia plicata en la zona de los T-5 al T-10, (Mújica, inédito), mientras que Dendrophylax lindenii (D1) y Pleurothallis caymanense (Pca) presentan los valores más bajos con solo un individuo localizado. Las especies más comunes son B. cubensis y Encyclia sp., que aparecen en todos los transectos, así como Prosthechea boothia$n a(\mathrm{~Pb})$ que aparece en 6 de ellos; el resto, Broughtonia ortgiesiana (Bo), Tolumnia guibertiana (Tg), Tolumnia sp. (Tsp) y Polystachya concreta (Pco), es poco común, apareciendo de forma intermitente en los mismos.

Se debe destacar que como resultado de este estudio se hace el reporte de Broughtonia ortgiesiana por primera vez para Cabo San Antonio. Solo se detectó un individuo en flor por lo que no hay dudas de que se encuentra también en esta zona. Se le reportaba solo para la zona de La Bajada, en la confluencia de Cabo San Antonio y Cabo Corrientes a unos $50 \mathrm{~km}$. de este lugar. Hasta el momento se pensaba que $B$. cubensis y $B$. ortgiesiana no eran simpátricas.

Aunque B.c. es la especie más común con el $55,45 \%$ del total de individuos, esta no se distribuye por igual. Existen diferencias significativas en cuanto a su distribución en los transectos $\left(?^{2}=275,606\right.$, $\mathrm{p}=0.0001$. Mientras que en el T-4 encontramos 124 individuos, en el T-3 solo se localizaron 7 de ellos (Tabla 3). Este aspecto reafirma el patrón de fuerte agregación que muestra esta especie.

TABLA 3. Individuos de cada especie de orquídea presentes en cada transecto.

\begin{tabular}{r|r|r|r|r|r|r|r|r|r} 
& \multicolumn{10}{c}{ No de individuos de todas las especies de orquídeas por transecto } \\
\hline Transectos & \multicolumn{1}{|c}{ Bc } & Eb & Dl & Esp & Tg & Tsp & Pb & Pca & Pco \\
\hline 1 & 18 & 5 & 0 & 18 & 22 & 2 & 0 & 0 & 0 \\
\hline 2 & 61 & 0 & 0 & 7 & 2 & 28 & 0 & 0 & 0 \\
\hline 3 & 7 & 1 & 0 & 4 & 0 & 0 & 2 & 1 & 0 \\
\hline 4 & 124 & 0 & 0 & 5 & 0 & 0 & 0 & 0 & 0 \\
\hline 5 & 11 & 0 & 0 & 44 & 2 & 0 & 5 & 0 & 0 \\
\hline 6 & 29 & 0 & 1 & 20 & 0 & 0 & 4 & 0 & 0 \\
\hline 7 & 50 & 0 & 0 & 23 & 0 & 0 & 4 & 0 & 0 \\
\hline 8 & 33 & 0 & 0 & 38 & 0 & 0 & 0 & 0 & 0 \\
\hline 9 & 19 & 0 & 0 & 24 & 0 & 0 & 3 & 0 & 0 \\
\hline 10 & 34 & 0 & 0 & 35 & 0 & 0 & 6 & 0 & 4
\end{tabular}


ANALISIS DE RIQUEZA-ABUNDANCIA EN LOS 10 TRANSECTOS - La riqueza de especies de orquídeas no sufre cambios drásticos de un transecto a otro por lo que no existen diferencias significativas en la misma $\left(\chi^{2}=2.889, \mathrm{p}=0.969\right)$. Un total de 6 transectos muestran una gran similitud en cuanto al número de especies encontradas en ellos.

Sin embargo, cuando analizamos la abundancia de las diferentes especies en los transectos encontramos diferencias significativas $\left(\chi^{2}=119.71, \mathrm{p}=0.0001\right)$. El más abundante resulta el T-4, por la dominancia de B.c., mientras que el menos abundante resultó el T-3, con solo 15 individuos. Este último es, sin embargo, uno de los transectos que muestra mayor equitatividad pues los 15 individuos pertenecen a 5 especies diferentes.

Por su parte, el T-4, siendo el de mayor abundancia, es uno de los dos transectos menos equitativo pues solo están presentes dos especies, todo debido, como hemos indicado antes, a la dominancia marcada de B.c.

ESTUdio DEL PATRON DE AGREGACION DE LA ESPECIE Para poder estudiar el patrón de agregación de la especie se ha comparado el porcentaje de individuos de B.c. creciendo solo o agregado en un radio de 10 $\mathrm{cm}$. de cada ejemplar. Se hace un estudio de este patrón para poder construir una matriz con individuos creciendo en solitario y otra con individuos creciendo agregados y así estudiar el posible efecto de la competencia sobre otras variables en el futuro. La figura 8 muestra el porcentaje de individuos creciendo solos y agregados. Se encontraron diferencias significativas según las formas de crecimiento $\left(\chi^{2}=4.167, \mathrm{p}=0.041\right)$. Es más frecuente estar agregado, o sea con otros individuos en el radio de $10 \mathrm{~cm}$. de distancia.

Competencia - Después de conocer que es más frecuente crecer agregado a otros individuos que solo quisimos conocer cual o cuales eran las especies más comunes de estos individuos en competencia con B.c. en el radio antes señalado. Se puede comprobar en la figura 9, que la especie vecina más frecuente es ella misma $\left(\chi^{2}=711.31, \mathrm{p}=0.0001\right)$. Lo cual parece indicar una fuerte competencia intraespecífica.

ANÁlisis ESPACIAL DE LAS ClASES DE VIDA DE LA POBLACION DE BRoughtonia CUBENSIS (Lindl.) Cogn. - Se ha analizado la estructura en clases de vida de la población monitoreada. Como se ha apuntado ante-

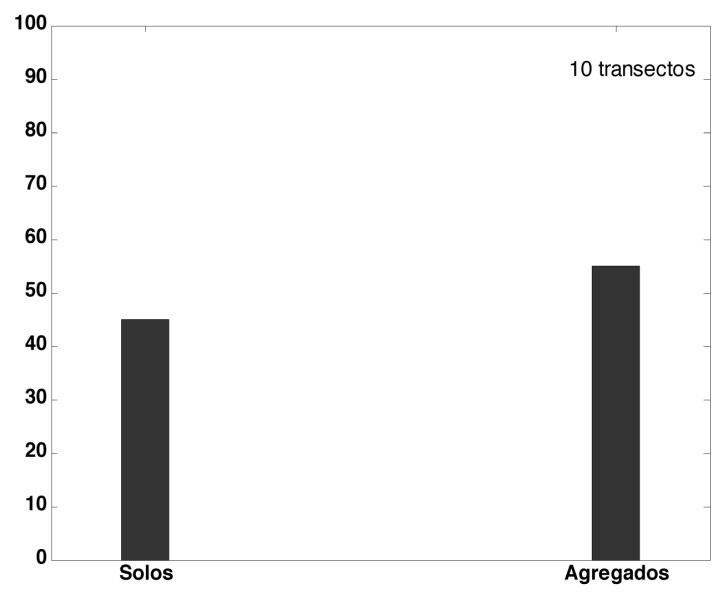

Figura 8. Por ciento de individos aislados y agregados.

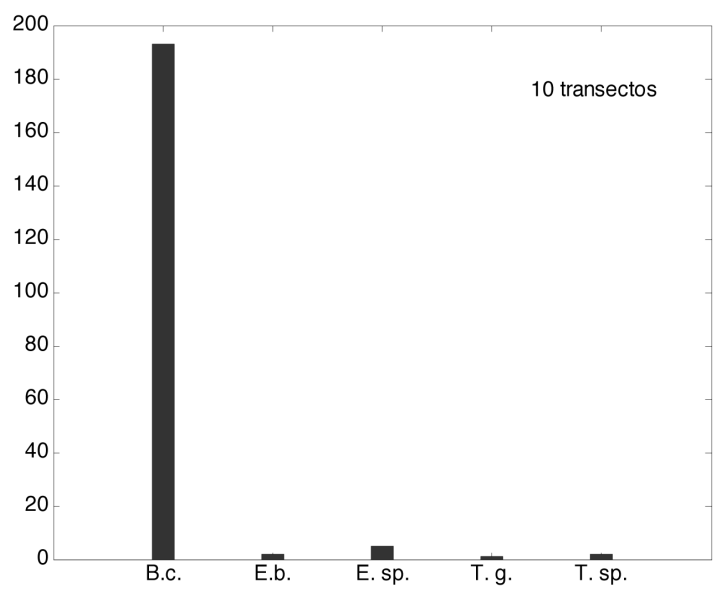

FIgURA 9. Competencia en B. cubensis.

riormente, en la misma se han censado y debidamente marcado un total de 365 individuos de B.c. tanto en condiciones aisladas como agregadas.

En la estructura actual en clases de vida (figura 10) para condiciones agregadas y en condiciones solitarias, se observa claramente que en la $\mathrm{C}-1$, no existen diferencias marcadas $\left(\chi^{2}=0.333, \mathrm{p}=0.564\right)$. entre el número de individuos en solitario (40) y los que se encuentran agregados (35), la especie en ese primer estadio no tiene preferencias por estar en solitario o en condiciones agregadas. Igualmente se puede distinguir, que en las $\mathrm{C}-2\left(\chi^{2}=0.514\right.$, $\mathrm{p}=0.473)$ y $\mathrm{C}-3\left(\chi^{2}=2.809, \mathrm{p}=0.094\right)$ las diferencias no son significativas. Sin embargo, en la C-4 se observa que la especie prefiere estar agregada. $\left(\chi^{2-}\right.$ $=4.129, \mathrm{p}=0.042$ ). 


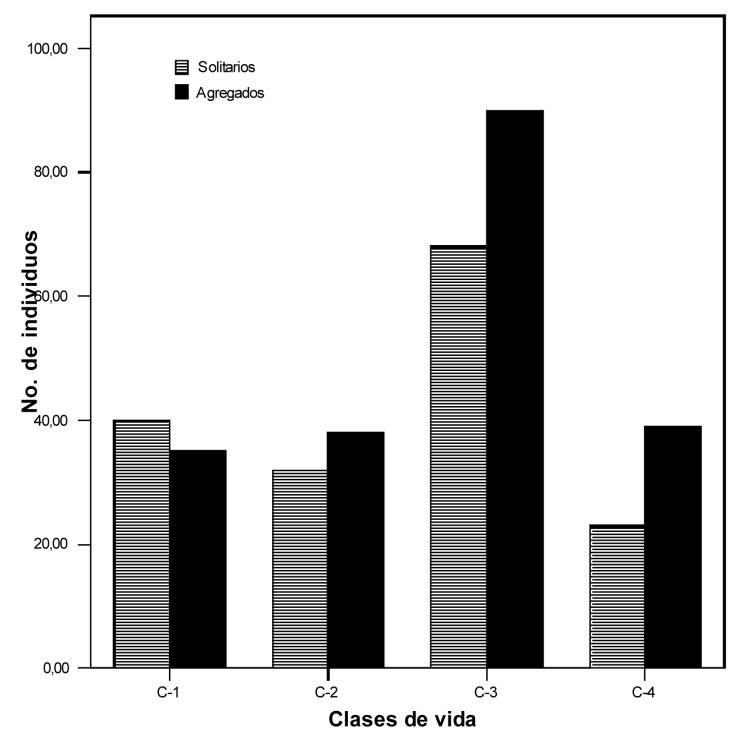

Figura 10. Número de individuos en cada clase de vida en condiciones solitarias o agregadas.

\section{Discusión}

La Reserva de la Biosfera de la Península de Guanahacabibes es un conjunto de frágiles ecosistemas en los cuáles conviven un sinnúmero de especies tanto vegetales como animales. La familia Orchidaceae está ampliamente representada en la misma. Los últimos estudios realizados y publicados indicaban la presencia de 28 especies de esta familia (Ferro et al. 1995). De acuerdo a los estudios que actualmente se realizan en esta península por los investigadores del Orquideario Soroa, el número ya llega a 34 especies diferentes, contándose con 6 especies endémicas de esta zona, de ellas 2 endémicos locales. Aunque en algunos momentos se han hecho estudios sobre el hábitat de algunas de estas especies (Ferro, inédito) estos no se han realizado con el objetivo de conocer sobre la compleja dinámica de las diferentes clases de vida que componen las poblaciones que allí existen y que se asientan, en muchos casos, en diferentes ecosistemas.

Broughtonia cubensis ocupa la porción más oeste del Cabo San Antonio, extendiéndose a lo largo de una larga franja costera ocupando lo mismo el lindero del bosque semideciduo que el matorral xeromorfo costero. Prefiere los ambientes abiertos, recibiendo la mayoría de las plantas una fuerte dosis de insolación a baja y mediana altura (Mújica, inédito), prefiriendo el matorral xeromorfo costero al bosque semideciduo. La comunidad de forofitos ocupados es diversa, sin embargo, se observa una preferencia por Plumeria tuberculata y Erythroxylum areolatum. Entre ambas representan el $60 \%$ de todos los forofitos ocupados. Otras especies sumamente abundantes en el área estudiada presentaron sin embargo bajos porcentajes de ocupación.

$\mathrm{Al}$ analizar las similitudes o diferencias entre transectos, de acuerdo a las especies de forofitos ocupados, se observó que Guettarda calyptrata, es la especie de forofito que obtuvo el $10,4 \%$ del total de individuos de la población monitoreada concentrado en un solo individuo, encontrándose otros forofitos de la misma especie sin ningún individuo de B.c., lo cual vuelve a incidir en el carácter agregado de esta orquídea no sólo a nivel de especies de forofitos sino del individuo dentro de una especie de forofito dada. Este resultado va en el mismo sentido al apuntado por Steege and Cornelissen (1989), Brown (1990) y Waechter (1998) que encuentran que las epífitas, usualmente se distribuyen de forma irregular a lo largo de los forofitos, presentando una variación vertical, tanto en número de individuos como de especies.

En la estructura espacial se demuestra que existen diferencias significativas en cuanto a crecer aislado o en agregación, prefiriendo esta última. Refiriéndonos a las clases de vida en particular se constata que solo la clase 4 o adultos reproductores de mayor tamaño tiene una preferencia por vivir en forma agregada, marcando la diferencia a nivel de especie. Esta estructura de agregación de los individuos mayores puede ser el resultado de la competencia entre plántulas durante el desarrollo de la población.

La especie en estudio prefiere la orientación hacia el norte y el este, posiblemente debido a que en la mayor parte del año los vientos tienen un componente que va del norte al este. Existe una competencia intraespecífica fuerte pues existen diferencias significativas en cuanto a los competidores, es raro encontrar individuos de otras especies en competencia con B.c., el principal competidor por el espacio es ella misma.

La corteza ha sido uno de los temas de discusión respecto a su utilización como indicador en los estudios del epifitismo. Los debates se han centrado en las causas de su importante papel, pudiéndose extraer que hay dos elementos básicos: su estructura física (tipo de corteza) como sustrato de fijación, y su composición, como suministrador de los elementos quí- 
micos necesarios. Hilitzer (1925) citado por Barkman (1959) denegó la influencia de los factores químicos, y consideró las diferencias que produce el relieve de la corteza. Cuello (1998) señala que para las epífitas, como para cualquier otro organismo sésil, las características del sustrato, especialmente durante su establecimiento, son particularmente importantes y resalta dentro de éstas el tipo de superficie de la corteza de los forofitos.

Algunos de los factores que pueden influenciar los patrones de distribución y abundancia de epífitas vasculares en los bosques tropicales, se relacionan, entre otras, con la arquitectura, porte y características de la corteza de los forofitos (Lüttge 1989, Steege \& Cornelissen 1989).

B.c. se encuentra preferentemente sobre corteza lisa o rugosa, debido al tipo de especie escogida como huésped, Plumeria tuberculata (lisa) y Erythroxylum areolatum (rugosa). El hecho de preferir los extremos (lisa o rugosa) y no el término medio (semirugosa) pone en duda las consideraciones de los autores antes mencionados. Nuestra hipótesis se basa en que la especie no elige un tipo de corteza determinado, sino que coloniza aquellos espacios con presencia de micorrizas, sin las cuáles es imposible el surgimiento y establecimiento de nuevos individuos. Reafirma esta hipótesis el hecho de que en un área pequeña podemos encontrar forofitos de la misma especie con un sinnúmero de individuos habitando sobre sí, mientras que otros de igual especie a 1-2 metros de distancia no resultan ocupados. Este fenómeno está siendo objeto de estudio actualmente (Mújica, inédito).

Hay en general una dominancia de B.c. con relación a las otras especies a lo largo de todos los transectos. Esta dominancia implica una agregación de individuos de esta especie que hace probable que la competencia intraespecífica sea una de las fuerzas que condicionan la estructura y dinámica de esta población. En la literatura se cita frecuentemente que las especies de orquídeas epífitas se suelen encontrar de forma agregada (Ackerman 1995). El análisis de las formas de vida para B.c. nos ha permitido analizar esto con detalle. Las semillas de esta especie de orquídea son muy pequeñas y se diseminan en el espacio con igual probabilidad en todas las direcciones. Esto hace que la distribución más probable sea una distribución al azar y no agregada de las plántulas. Sin embargo, no todos los sitios tienen la misma calidad. Esta calidad está asociada probablemente a la presencia de micorrizas, hipótesis con las que estamos actualmente trabajando, esto a su vez implica una competencia por los mejores sitios que se manifiesta en que los individuos más grandes, adultos reproductivos C-4, puedan controlar los sitios de "mayor calidad".

Nuestros resultados parecen indicar que puede existir una zona núcleo de dispersión para esta especie localizada en el área comprendida en el transecto T-4 entre el bosque semideciduo y la vegetación de pantano. Este resultado debido, a la estructura metapoblacional de esta especie, debería tener importantes implicaciones para el planteamiento y manejo de las estrategias de conservación dentro de la región Caribeña que es un 'biodiversity hotspot' (Myers et al. 2000). Un excelente estudio de bases propiamente metapoblacionales se encuentra en Tremblay et al. (2006), donde los autores analizan la idoneidad del modelo metapoblacional de Levins para las especies de un orquídea tropical epifita del género Lepanthes.

Agradecimientos. Los autores de este trabajo desean expresar su agradecimiento a todas las personas que de una forma u otra apoyaron su realización. De modo muy especial al Dr. Jorge Ferro Díaz, Director de ECOVIDA en la provincia de Pinar del Río, por el apoyo incondicional, sin el cual hubiera sido imposible la realización del mismo. A los compañeros de la Estación Meteorológica del Cabo San Antonio, de la Estación Ecológica de la Península de Guanahacabibes, a los trabajadores de ALMEST Ena y Santiago, a Sonia Maña Alvarenga que vino desde Alicante, España, a soportar los rigores de nuestro clima y colaborar en los monitoreos. A los torreros del Faro Roncali, Felicia, Felino, Luis y Pipo, por la asistencia y apoyo en todo momento. A todos los colegas del Jardín Botánico Orquideario Soroa, por ayudarnos desinteresadamente en los monitoreos.

\section{LITERATURA CITADA}

Ackerman, J.D. 1995. An Orchid Flora of Puerto Rico and The Virgen Islands. The New York Botanical Garden. U.S.A.

Barkman, J. J. 1959. Phytosociology and ecology of cryptogamic epiphytes. Van Gorcum \& Co., Assen, Netherlands.

Begoña, B.A. 2000. Biología de la Conservación de plantas amenazadas. Organismo Autónomo Parques Nacionales. Madrid. España. 
Brown, D.A. 1990. El epifitismo en las selvas montanas del Parque Nacional "El Rey" Argentina: Composición florística y patrón de distribución. Rev. Biol.Trop. 38: 155-166.

Cuello, A. 1998. A review of sampling procedures for the study of vascular epiphytic species diversity in neotropical montane forest. Herbario Universitario PORT, Programa de Recursos Naturales Renovables, UNELLEZ-Guanare, Venezuela.

Díaz, M.A. 1996. Las orquídeas de Cuba. Editorial Científico Técnica. La Habana, Cuba.

Delgado, F.F. 2000. Funcionamiento de bosques semideciduos y caracterización de otros ecosistemas terrestres en la Reserva de la Biosfera Península de Guanahacabibes, Cuba. Informe Final Proyecto 01307029 PNCT "Los Cambios Globales y la Evolución del Medio Ambiente en Cuba". Agencia de Ciencia y tecnología. CITMA. Pinar del Río.

Ferro Díaz. J. et al. 1995. Mapa de Vegetación actual de la Reserva de la Biosfera Península de Guanahacabibes, Pinar del Río, Cuba. 1:100000. Memorias del II
Simposio Internacional HUMEDALES'94. Ciénaga de Zapata, Septiembre de 1994. Editorial Academia.

Herrera, M. et al. 1987. Las Reservas de la Biosfera en Cuba. Instituto de Ecología y Sistemática, Academia de Ciencias de Cuba.

Hutchings, M. J. 1989. Conservation and the British orchid flora. Plants Today 2: 50-58.

Kindlmann, P., J.H. Willems \& D.F. Whigham. 2002. Trends and Fluctuations and Underlying Mechanisms in Terrestrial Orchid Populations. Backhuys Publishers, Leiden.

Kull, T. 2002. Population dynamics of north temperate orchids. In: T. Kull \& J. Arditti (eds.), Orchid Biology: Review and Perspectives, vol. VIII. Kluwer Academic Publishers, Dordrecht, The Netherlands.

Leyva Pagán, G. 2002. Guanahacabibes, donde se guarda el sol de Cuba. Editorial Academia, La Habana.

Lopetegui, C.M. et al. 1999. Caracterización climática y bioclimática de la Península de Guanahacabibes. Memorias del Taller Internacional TROPICO'99. Instituto de Meteorología. C 1989. 